\title{
Gap functions for quasi-variational inequalities via duality
}

\section{Altangerel ${ }^{*}$ (B)}

"Correspondence:

altangere@@gmit.edu.mn German-Mongolian Institute for Resources and Technology, Nalaikh, Mongolia

\section{Springer}

\begin{abstract}
This paper deals with an application of duality theory in optimization to the construction of gap functions for quasi-variational inequalities. The same approach was investigated for variational inequalities and equilibrium problems in (Pac. J. Optim. 2(3): 667-678, 2006; Asia-Pac. J. Oper. Res. 24(3): 353-371, 2007), and the study shows that we can obtain some previous results for variational inequalities as special cases. Moreover, some applications dealing with the generalized Nash equilibrium problems and mixed variational inequalities are presented.
\end{abstract}

\section{Introduction}

The quasi-variational inequality which is a generalization of the variational inequality problem was introduced first by Bensoussan et al. in [3] in the context of impulse control problems. The quasi-variational inequalities have many applications in economics, game theory, optimization and other applied sciences. It is well known that the generalized Nash equilibrium problem can be reduced to the quasi-variational inequality problem (see [4]).

A gap function approach is one of the main tools for solving variational inequalities. Different approaches on gap functions for quasi-variational inequalities have been investigated by various authors [5-9]. On the other hand, by using different dual problems in convex optimization (see $[10,11]$ ), gap functions for variational inequalities and equilibrium problems have been investigated in [1,2]. Specially, in [2], based on the conjugate duality for optimization problem, some gap functions for mixed variational inequalities, also dual gap functions for the variational inequality and the relation between these functions have been investigated. However, it still remains an open question how the same approach can be extended to quasi-variational inequalities. This paper aims to answer this question by applying duality results from [12] which deals with minimization of a convex function over the solution set of a range inclusion problem determined by a set-valued mapping.

The paper is organized as follows. Section 2 deals with some preliminary results from [12]. In Section 3 we consider the duality based approach on gap functions for quasivariational inequalities. Section 4 is devoted to the investigation of gap functions for mixed quasi-variational inequalities. Finally, some applications dealing with mixed variational inequalities and the generalized Nash equilibrium problems are presented in Section 5.

(c) The Author(s) 2018. This article is distributed under the terms of the Creative Commons Attribution 4.0 International License (http://creativecommons.org/licenses/by/4.0/), which permits unrestricted use, distribution, and reproduction in any medium, provided you give appropriate credit to the original author(s) and the source, provide a link to the Creative Commons license, and indicate if changes were made. 


\section{Preliminaries}

We consider the following optimization problem:

(P) $\inf _{0 \in F(x)} f(x)$,

where $f: \mathbb{R}^{n} \rightarrow \overline{\mathbb{R}}$ is a given function and $F: \mathbb{R}^{n} \rightrightarrows \mathbb{R}^{n}$ is a set-valued mapping such that $\operatorname{dom} f \cap F^{-1}(0) \neq \varnothing$.

Then the corresponding dual problem becomes

$$
\text { (D) } \sup _{p \in \mathbb{R}^{n}} \inf _{x \in \mathbb{R}^{n}}\left[f(x)+s_{F}(x, p)\right]
$$

where $s_{F}: \mathbb{R}^{n} \times \mathbb{R}^{n} \rightarrow \overline{\mathbb{R}}$ is the lower support function associated with $F$ defined by

$$
s_{F}(x, p)=\inf _{y \in F(x)}\langle p, y\rangle
$$

and $\langle\cdot, \cdot\rangle$ is the Euclidean inner product.

Proposition 1 ([12]) Let $f: \mathbb{R}^{n} \rightarrow \overline{\mathbb{R}}$ be a proper and convex function and $F: \mathbb{R}^{n} \rightrightarrows \mathbb{R}^{n}$ be a convex set-valued mapping. If the constraint qualification

(CQ) $\exists \bar{x} \in \operatorname{ri}(\operatorname{dom} f) \cap \operatorname{ri}(\operatorname{dom} F)$ and $0 \in \operatorname{ri}(F(\bar{x}))$

is fulfilled, then for $(P)$ and $(D)$ strong duality holds, i.e., $\exists \bar{p} \in \mathbb{R}^{n}$ such that

$$
\begin{aligned}
\inf _{0 \in F(x)} f(x) & =\sup _{p \in \mathbb{R}^{n}} \inf _{x \in \mathbb{R}^{n}}\left[f(x)+s_{F}(x, p)\right] \\
& =\inf _{x \in \mathbb{R}^{n}}\left[f(x)+s_{F}(x, \bar{p})\right],
\end{aligned}
$$

where $\operatorname{dom} h$ is the effective domain of a given function $h: \mathbb{R}^{n} \rightarrow \overline{\mathbb{R}}$ and $\mathrm{ri}(C)$ is the relative interior of a given set $C \subseteq \mathbb{R}^{n}$.

\section{Gap function for quasi-variational inequalities}

Let $T: \mathbb{R}^{n} \rightarrow \mathbb{R}^{n}$ be a continuous vector-valued function and $K: \mathbb{R}^{n} \rightrightarrows \mathbb{R}^{n}$ be a set-valued mapping such that $K(x)$ is nonempty, closed and convex for each $x \in \mathbb{R}^{n}$. Then the quasivariational inequality problem consists in finding a vector $x \in K(x)$ such that

$$
(Q V I) \quad\langle T(x), y-x\rangle \geq 0, \quad \forall y \in K(x) .
$$

For a fixed $x \in \mathbb{R}^{n},(Q V I)$ can be rewritten as an optimization problem

$$
\left(P^{Q V I} ; x\right) \quad \inf _{y \in K(x)}\langle T(x), y-x\rangle .
$$

Let us define a function $\gamma^{Q V I}(x): \mathbb{R}^{n} \rightarrow \overline{\mathbb{R}}$ for $x \in \mathbb{R}^{n}$ (cf. [2])

$$
\gamma^{Q V I}(x):=-v\left(D^{Q V I} ; x\right)
$$


where $v\left(D^{Q V I} ; x\right)$ denotes the optimal objective value of the dual problem for $\left(P^{Q V I} ; x\right)$. Since $\left(P^{Q V I} ; x\right)$ can be reformulated as

$$
\inf _{0 \in K(x)-y}\langle T(x), y-x\rangle
$$

the dual problem for $\left(P^{Q V I} ; x\right)$ turns out to be

$$
\begin{aligned}
\left(D^{Q V I} ; x\right) \quad \sup _{p \in \mathbb{R}^{n}} \inf _{y \in \mathbb{R}^{n}}\left[\langle T(x), y-x\rangle+s_{K(x)-i d}(y, p)\right] \\
\quad=\sup _{p \in \mathbb{R}^{n}} \inf _{y \in \mathbb{R}^{n}}\left[\langle T(x), y\rangle+s_{K(x)-i d}(y, p)\right]-\langle T(x), x\rangle .
\end{aligned}
$$

Consequently, we have

$$
\gamma^{Q V I}(x)=\langle T(x), x\rangle-\sup _{p \in \mathbb{R}^{n}} \inf _{y \in \mathbb{R}^{n}}\left[\langle T(x), y\rangle+s_{K(x)-i d}(y, p)\right] .
$$

Let us recall now the definition of a gap function for quasi-variational inequalities and give an auxiliary result.

Definition 1 A function $\gamma: \mathbb{R}^{n} \rightarrow \overline{\mathbb{R}}$ is said to be a gap function for the problem $(Q V I)$ if it satisfies the following properties:

(i) $\gamma(y) \geq 0, \forall y \in K(x)$;

(ii) $\gamma(x)=0$ if and only if $x$ solves the problem $(Q V I)$.

Lemma 1 Let $K: \mathbb{R}^{n} \rightrightarrows \mathbb{R}^{n}$ be a set-valued mapping and $p \in \mathbb{R}^{n}$ be fixed. Then, for any $x \in \mathbb{R}^{n}$, it holds

$$
s_{K(x)-i d}(y, p)=s_{K}(x, p)-\langle p, y\rangle .
$$

Proof Let $x \in \mathbb{R}^{n}$ and $p \in \mathbb{R}^{n}$ be fixed. Then, by definition, we have

$$
\begin{aligned}
& s_{K(x)-i d}(y, p)=\inf _{z \in K(x)-y}\langle p, z\rangle \\
& (z+y:=t)=\inf _{t \in K(x)}\langle p, t-y\rangle=\inf _{t \in K(x)}\langle p, t\rangle-\langle p, y\rangle=s_{K}(x, p)-\langle p, y\rangle .
\end{aligned}
$$

Proposition 2 For the problem $(Q V I)$, we have

$$
\gamma^{Q V I}(x)= \begin{cases}-\inf _{y \in F(x)}\langle T(x), y-x\rangle, & p=T(x), \\ +\infty, & \text { otherwise }\end{cases}
$$

Proof By using Lemma 1, one obtains that

$$
\begin{aligned}
\gamma^{Q V I}(x) & =\langle T(x), x\rangle-\sup _{p \in \mathbb{R}^{n}} \inf _{y \in \mathbb{R}^{n}}\left[\langle T(x), y\rangle+s_{K(x)-i d}(y, p)\right] \\
& =\langle T(x), x\rangle-\sup _{p \in \mathbb{R}^{n}}\left[s_{K}(x, p)+\inf _{y \in \mathbb{R}^{n}}\langle T(x)-p, y\rangle\right] .
\end{aligned}
$$


From

$$
\inf _{y \in \mathbb{R}^{n}}\langle T(x)-p, y\rangle= \begin{cases}0, & T(x)-p=0 \\ -\infty, & \text { otherwise }\end{cases}
$$

it follows that

$$
\begin{aligned}
\gamma^{Q V I}(x) & = \begin{cases}\langle T(x), x\rangle-\sup _{p \in \mathbb{R}^{n}} s_{K}(x, T(x)), & p=T(x), \\
+\infty, & \text { otherwise }\end{cases} \\
& = \begin{cases}\langle T(x), x\rangle-\inf _{y \in F(x)}\langle T(x), y\rangle, & p=T(x), \\
+\infty, & \text { otherwise }\end{cases} \\
& = \begin{cases}-\inf _{y \in F(x)}\langle T(x), y-x\rangle, & p=T(x), \\
+\infty, & \text { otherwise. }\end{cases}
\end{aligned}
$$

Remark 1 A gap function $\gamma(x)=-\inf _{y \in F(x)}\langle T(x), y-x\rangle$ was investigated in [7] (see also [6]).

\section{Gap functions for mixed quasi-variational inequalities}

Let $T: \mathbb{R}^{n} \rightarrow \mathbb{R}^{n}$ be a continuous vector-valued function and $K: \mathbb{R}^{n} \rightrightarrows \mathbb{R}^{n}$ be a set-valued mapping such that $K(x)$ is nonempty, closed and convex for each $x \in \mathbb{R}^{n}$. Let $\varphi: \mathbb{R}^{n} \rightarrow \overline{\mathbb{R}}$ be a given function. Then the mixed quasi-variational inequality problem consists in finding a vector $x \in K(x)$ such that (cf. [13])

$$
(M Q V I) \quad\langle T(x), y-x\rangle+\varphi(y)-\varphi(x) \geq 0, \quad \forall y \in K(x)
$$

Rewriting $(M Q V I)$ as an optimization problem

$$
\left(P^{M Q V I} ; x\right) \quad \inf _{y \in K(x)}[\langle T(x), y-x\rangle+\varphi(y)-\varphi(x)]
$$

and repeating the same techniques in Section 3, we can define the following function:

$$
\gamma^{M Q V I}(x)=-\sup _{p \in \mathbb{R}^{n}} \inf _{y \in \mathbb{R}^{n}}\left[\langle T(x), y-x\rangle+\varphi(y)-\varphi(x)+s_{K(x)-i d}(y, p)\right] .
$$

It can be rewritten as

$$
\begin{aligned}
\gamma^{M Q V I}(x) & =\langle T(x), x\rangle+\varphi(x)-\sup _{p \in \mathbb{R}^{n}} \inf _{y \in \mathbb{R}^{n}}\left[\langle T(x), y\rangle+\varphi(y)+s_{K}(x, p)-\langle p, y\rangle\right] \\
& =\langle T(x), x\rangle+\varphi(x)+\inf _{p \in \mathbb{R}^{n}}\left[-s_{K}(x, p)-\inf _{y \in \mathbb{R}^{n}}[\langle T(x)-p, y\rangle-\varphi(y)]\right. \\
& =\langle T(x), x\rangle+\varphi(x)+\inf _{p \in \mathbb{R}^{n}}\left[-s_{K}(x, p)+\varphi^{*}(p-T(x))\right],
\end{aligned}
$$

where $h^{*}: \mathbb{R}^{n} \rightarrow \overline{\mathbb{R}}$ is the conjugate function of a given function $h: \mathbb{R}^{n} \rightarrow \overline{\mathbb{R}}$ defined by $h^{*}(p)=\sup _{x \in \mathbb{R}^{n}}[\langle p, x\rangle-h(x)]$. 
Theorem 1 Let $K: \mathbb{R}^{n} \rightrightarrows \mathbb{R}^{n}$ be a set-valued mapping such that $K(x)$ is nonempty, closed and convex for each $x \in \mathbb{R}^{n}$ and $\varphi$ be convex. If, for each $x \in \mathbb{R}^{n}$, the constraint qualification

$$
(C Q ; x) \quad \exists \bar{x} \in \operatorname{ri}(\operatorname{dom} \varphi) \quad \text { and } \quad 0 \in \operatorname{ri}(K(x)-\bar{x})
$$

is fulfilled, then $\gamma^{M Q V I}$ is a gap function for (MQVI).

Proof

(i) Let $x \in \mathbb{R}^{n}$ be fixed. By weak duality, one gets

$$
v\left(D^{M Q V I}, x\right) \leq v\left(P^{M Q V I}, x\right) \leq 0
$$

where $\left(D^{M Q V I} ; x\right)$ is a dual problem for $\left(P^{M Q V I} ; x\right)$. Consequently, we have $\gamma^{M Q V I}(x)=-v\left(D^{M Q V I}, x\right) \geq 0$.

(ii) If $\gamma^{M Q V I}(x)=0$, then

$$
0=v\left(D^{M Q V I}, x\right) \leq v\left(P^{M Q V I}, x\right) \leq 0 .
$$

In other words, $v\left(P^{M Q V I}, x\right)=0$, which means that $x$ is a solution of $(M Q V I)$.

Conversely, if $x$ is a solution to the problem $(M Q V I)$, then $v\left(P^{M Q V I}, x\right)=0$. By

Proposition 1, we obtain that

$$
\gamma^{M Q V I}(x)=-v\left(D^{M Q V I}, x\right)=-v\left(P^{M Q V I}, x\right)=0 .
$$

Remark 2 Let us assume that $T: \mathbb{R}^{n} \rightarrow \mathbb{R}^{n}$ is affine and $K: \mathbb{R} \rightrightarrows \mathbb{R}^{n}$ is a set-valued mapping. It is easy to check that $s_{K}(x, p)$ is concave with respect to $p$. By assumption, $\varphi^{*}(p-T(x))$ is convex, and therefore, the last term in $\gamma^{M Q V I}$ is a convex optimization problem for fixed $x \in \mathbb{R}^{n}$ :

$$
\inf _{p \in \mathbb{R}^{n}}\left[-S_{K}(x, p)+\varphi^{*}(p-T(x))\right]
$$

Remark 3 Let $K(x) \equiv K$. Then we have the mixed variational inequality which consists in finding a vector $x \in K$ such that

$(M V I) \quad\langle T(x), y-x\rangle+\varphi(y)-\varphi(x) \geq 0, \quad \forall y \in K$.

In this case, we have $s_{K}(x, p)=-\delta_{K}^{*}(-p)$, where $\delta_{K}: \mathbb{R}^{n} \rightarrow \overline{\mathbb{R}}$

$$
\delta_{K}(y)= \begin{cases}0, & \text { if } y \in K \\ +\infty, & \text { otherwise }\end{cases}
$$

denotes the indicator function of the set $K$. Consequently, one obtains

$$
\gamma^{M V I}(x)=\langle T(x), x\rangle+\varphi(x)+\inf _{p \in \mathbb{R}^{n}}\left[\delta_{K}(-p)+\varphi^{*}(p-T(x))\right],
$$

which is nothing else than the one investigated in [2]. 


\section{Special cases}

\subsection{The generalized Nash equilibrium problems}

The generalized Nash equilibrium problem (GNEP for short) is an extension of the classical Nash equilibrium problem, in which each player's strategy set depends on the rival player's strategies. We refer to [14] for the excellent comprehensive surveys on theories and algorithms for GNEP. We consider $N$ player's game. Each player $k$ controls his decision variable $x^{k} \in \mathbb{R}^{n_{k}}, n_{k} \in \mathbb{N}$, such that the vector $x=\left(x^{1}, \ldots, x^{N}\right) \in \mathbb{R}^{n}$ with $n=n_{1}+n_{2}+\cdots+n_{N}$ describes the decision vector of all players. We oft use the notation $x=\left(x^{k}, x^{-k}\right)$, where $x^{-k}=\left(x^{1}, x^{2}, \ldots, x^{k-1}, x^{k+1}, \ldots, x^{N}\right)$. Furthermore, each player $k$ has a cost function $\theta_{k}: \mathbb{R}^{n} \rightarrow \mathbb{R}$ and a strategy set $X_{k}\left(x^{-k}\right) \subseteq \mathbb{R}^{n_{k}}$ defined by the set-valued mapping $X_{k}: \mathbb{R}^{n-n_{k}} \rightrightarrows \mathbb{R}^{n_{k}}$. Let

$$
\Omega(x):=X_{1}\left(x^{-1}\right) \times \cdots \times X_{N}\left(x^{-N}\right) .
$$

Then $(G N E P)$ consists in finding a vector $\bar{x}=\left(\bar{x}^{1}, \ldots, \bar{x}^{N}\right)$ such that, for each $k=\overline{1, N}$, the vector $\bar{x}^{k}$ solves

$$
P_{k}\left(\bar{x}^{-k}\right) \inf _{x^{k} \in X_{k}\left(\bar{x}^{-k}\right)} \theta\left(x^{k}, \bar{x}^{-k}\right) .
$$

We assume that $X_{k} \subseteq \mathbb{R}^{n_{k}}, k=1, \ldots, N$, are nonempty, closed and convex sets and, for each fixed $x^{-k} \in \mathbb{R}^{n-n_{k}}$, the functions $\theta_{k}\left(\cdot, x^{-k}\right)$ are convex and differentiable.

Let us define the vector-valued function $F: \mathbb{R}^{n} \rightarrow \mathbb{R}^{n}$ by

$$
F(x)=\left(\nabla_{x^{k}} \theta_{k}\left(x^{k}, x^{-k}\right)\right)_{k=1}^{N}
$$

Then it is well known that (see [4]) (GNEP) is reduced to the problem of finding a vector $\bar{x} \in \Omega(\bar{x})$ such that

$$
\langle F(\bar{x}), y-\bar{x}\rangle \geq 0, \quad \forall y \in \Omega(\bar{x}) .
$$

Proposition 3 (cf. [12]) Assume that $\exists \bar{x} \in K(\bar{x})$ and $\bar{p} \in \mathbb{R}^{n}$ satisfying the following conditions:

(i) $\sum_{k=1}^{N}\left\langle\nabla_{x^{k}} \theta_{k}\left(\bar{x}^{k}, \bar{x}^{-k}\right), \bar{x}^{k}\right\rangle=\inf _{y \in \mathbb{R}^{n}}\left[\sum_{k=1}^{N}\left\langle\nabla_{x^{k}} \theta_{k}\left(\bar{x}^{k}, \bar{x}^{-k}\right), y^{k}\right\rangle+s_{\Omega(\bar{x})-i d}(y, \bar{p})\right]$;

(ii) $s_{\Omega(\bar{x})-i d}(\bar{x}, \bar{p})=0$.

Then $\bar{x}$ is a solution of (GNEP).

Proof Let $x \in K(x), p \in \mathbb{R}^{n}$ be fixed and conditions (i)-(ii) in Proposition 2 be fulfilled. Then, according to Lemma 1 , condition (i) can be rewritten as

$$
\begin{aligned}
& \sum_{k=1}^{N}\left\langle\nabla_{x^{k}} \theta_{k}\left(x^{k}, x^{-k}\right), x^{k}\right\rangle+s_{\Omega(x)-i d}(x, p) \\
& \quad=\inf _{y \in \mathbb{R}^{n}}\left[\sum_{k=1}^{N}\left\langle\nabla_{x^{k}} \theta_{k}\left(x^{k}, x^{-k}\right), y^{k}\right\rangle+s_{\Omega(x)-i d}(y, p)\right] \\
& \Leftrightarrow \sum_{k=1}^{N}\left\langle\nabla_{x^{k}} \theta_{k}\left(x^{k}, x^{-k}\right), x^{k}\right\rangle+s_{\Omega}(x, p)-\langle p, x\rangle
\end{aligned}
$$




$$
\begin{gathered}
=\inf _{y \in \mathbb{R}^{n}}\left[\sum_{k=1}^{N}\left\langle\nabla_{x^{k}} \theta_{k}\left(x^{k}, x^{-k}\right), y^{k}\right\rangle+s_{\Omega}(x, p)-\langle p, y\rangle\right] \\
\Leftrightarrow \quad \sum_{k=1}^{N}\left\langle\nabla_{x^{k}} \theta_{k}\left(x^{k}, x^{-k}\right)-p^{k}, x^{k}\right\rangle=\sum_{k=1}^{N} \inf _{y^{k} \in \mathbb{R}^{n_{k}}}\left\langle\nabla_{x^{k}} \theta_{k}\left(x^{k}, x^{-k}\right)-p^{k}, y^{k}\right\rangle \\
\Leftrightarrow \quad\left\langle\nabla_{x^{k}} \theta_{k}\left(x^{k}, x^{-k}\right)-p^{k}, x^{k}\right\rangle=\inf _{y^{k} \in \mathbb{R}^{n_{k}}}\left\langle\nabla_{x^{k}} \theta_{k}\left(x^{k}, x^{-k}\right)-p^{k}, y^{k}\right\rangle, \quad k=\overline{1, N .}
\end{gathered}
$$

From

$$
\inf _{y^{k} \in \mathbb{R}^{n} n^{n}}\left\langle\nabla_{x^{k}} \theta_{k}\left(x^{k}, x^{-k}\right)-p^{k}, y^{k}\right\rangle= \begin{cases}0, & \nabla_{x^{k}} \theta_{k}\left(x^{k}, x^{-k}\right)-p^{k}=0 \\ -\infty, & \text { otherwise }\end{cases}
$$

it follows that

$$
p^{k}=\nabla_{x^{k}} \theta_{k}\left(x^{k}, x^{-k}\right), \quad k=\overline{1, N}
$$

or, equivalently,

$$
p=F(x)
$$

Setting (2) in condition (ii), we obtain that

$$
\inf _{z \in \Omega(x)-x}\langle F(x), z\rangle=0
$$

which is nothing else than (1).

\subsection{Linear mixed variational inequality}

Recently, the following type of mixed variational inequality has been investigated intensively with particular interest in studying electrical circuits involving transistors (see [15] and [16]): to find a vector $x \in \mathbb{R}^{n}$ such that

$$
(L M V I) \quad\langle M x+q, y-x\rangle+\varphi(y)-\varphi(x) \geq 0, \quad \forall y \in \mathbb{R}^{n},
$$

where $M \in \mathbb{R}^{m \times n}$ is a real $P$-matrix and $q \in \mathbb{R}^{n}$. Since

$$
\delta_{\mathbb{R}^{n}}(-p)=\sup _{y \in \mathbb{R}^{n}}\langle-p, y\rangle= \begin{cases}0, & p=0 \\ +\infty, & \text { otherwise }\end{cases}
$$

we get

$$
\left.\gamma^{L M V I}(x)=\langle M x+q, x\rangle+\varphi(x)+\varphi^{*}(-M x-q)\right]
$$

By definition of a gap function,

$$
\gamma^{L M V I}(x)=0 \quad \Leftrightarrow \quad M x+q \in \partial \varphi(x) \quad \Leftrightarrow \quad 0 \in-M x-q-\partial \varphi(x) .
$$




\section{Acknowledgements}

The author is very grateful to Prof. R.I. Boţ and two anonymous referees for their valuable and helpful comments for improving the quality of the paper. The research funding was provided by the 'L2766-MON: Higher Education Reform' project financed by the Asian Development Bank and executed by the Ministry of Education, Culture, Science and Sports of Mongolia.

\section{Competing interests}

The author declares that they have no competing interests.

Authors' contributions

The author read and approved the final manuscript.

\section{Publisher's Note}

Springer Nature remains neutral with regard to jurisdictional claims in published maps and institutional affiliations.

Received: 24 October 2017 Accepted: 28 December 2017 Published online: 10 January 2018

\section{References}

1. Altangerel, L, Boț, RI, Wanka, G: On gap functions for equilibrium problems via Fenchel duality. Pac. J. Optim. 2(3), 667-678 (2006)

2. Altangerel, L, Boț, RI, Wanka, G: On the construction of gap functions for variational inequalities via conjugate duality. Asia-Pac. J. Oper. Res. 24(3), 353-371 (2007)

3. Bensoussan, A, Goursat, M, Lions, JL: Contrôle impulsionnel et inéquations quasivariationnelle. C. R. Acad. Sci. Paris, Sér. A 276, 1279-1284 (1973)

4. Harker, PT: Generalized Nash games and quasi-variational inequalities. Eur. J. Oper. Res. 54(1), 81-94 (1991)

5. Aussel, D, Correa, R, Marechal, M: Gap functions for quasivariational inequalities and generalized Nash equilibrium problems. J. Optim. Theory Appl. 151(3), 474-488 (2011)

6. Dietrich, H: A smooth dual gap function solution to a class of quasivariational inequalities. J. Math. Anal. Appl. 235, 380-393 (1999)

7. Fukushima, M: A class of gap functions for quasi-variational inequality problems. J. Ind. Manag. Optim. 3(2), 165-171 (2007)

8. Harms, N, Hoheisel, T, Kanzow, C: On a smooth dual gap function for a class of quasi-variational inequalities. J. Optim. Theory Appl. 163(2), 413-438 (2014)

9. Taji, K: On gap functions for quasi-variational inequalities. Abstr. Appl. Anal. 2008, Article ID 531361 (2008). https://doi.org/10.1155/2008/531361

10. Boț, Rl, Grad, SM, Wanka, G: Duality in Vector Optimization. Springer, Berlin (2009)

11. Wanka, G, Boţ, Rl: On the relations between different dual problems in convex mathematical programming. In: Chamoni, P, Leisten, R, Martin, A, Minnemann, J, Stadler, H (eds.) Operations Research Proceedings 2001, pp. 255-262. Springer, Berlin (2002)

12. Boț, Rl, Csetnek, ER: Conjugate duality and the control of linear discrete systems. J. Optim. Theory Appl. 159(3), 576-589 (2013)

13. Noor, MA: Some recent advances in variational inequalities, part II: other concepts. N.Z. J. Math. 26, 229-255 (1997)

14. Facchinei, F, Kanzow, C: Generalized Nash equilibrium problems. Ann. Oper. Res. 175, 177-211 (2010)

15. Addi, K, Despotovic, Z, Goeleven, D, Rodic, A: Modelling and analysis of a non-regular electronic circuit via variational inequality formulation. Appl. Math. Model. 35(5), 2172-2184 (2011)

16. Goeleven, D: Existence and uniqueness for a linear mixed variational inequality arising in electrical circuits with transistors. J. Optim. Theory Appl. 138(3), 397-406 (2008)

\section{Submit your manuscript to a SpringerOpen ${ }^{\circ}$ journal and benefit from:}

- Convenient online submission

- Rigorous peer review

- Open access: articles freely available online

- High visibility within the field

- Retaining the copyright to your article

Submit your next manuscript at $\downarrow$ springeropen.com 